\title{
Electromechanical Development of a Low Cost End Effector Pose Estimation System for Articulated Excavators
}

\author{
Kurt M. Lundeen ${ }^{a}$, Suyang Dong ${ }^{a}$, Nicholas Fredricks ${ }^{a}$, Manu Akula $^{a}$, and Vineet R. Kamat ${ }^{a}$ \\ ${ }^{a}$ Department of Civil and Environmental Engineering, University of Michigan, US \\ E-mail:klundeen@umich.edu,dsuyang@umich.edu,nfredricks@gmail.com, \\ akulaman@umich.edu,vkamat@umich.edu
}

\begin{abstract}
Vision-based pose estimation, in which optical cameras monitor fiducial markers to determine the three dimensional positioning and orientation of an articulated machine's end effector, offers a promising low-cost alternative to currently available sensor packages that are non-ubiquitous and cost prohibitive for a large portion of the market. Whereas traditional sensor systems determine end effector pose via kinematic chains passing through the links of a machine, optical sensor systems are capable of determining pose by observing an end effector directly. However, since markers cannot be mounted on an excavator's bucket for occlusion and durability reasons, a short kinematic chain must be used. An electromechanical design is proposed to provide such function for a low cost marker-based excavator pose estimation system. Several iterations of design and experimentation are discussed, including a four-bar linkage system, a synchronous belt system, a bucket linkage system, and a cable potentiometer system. The four-bar linkage and toothed belt systems were designed to transmit bucket angle information to cameras through the manipulation of a marker's pose, but were found to possess Gimbal lock and practicality issues, respectively. To overcome such issues, a generalized mapping approach was adopted and implemented in a bucket linkage design and a cable potentiometer design. The viability of the cable potentiometer system was experimentally confirmed, along with the identification of further work needed to refine the technology for large-scale practical implementation.

Keywords -

Camera-Marker Network; Machine Control; Pose Estimation; Construction Equipment Monitoring
\end{abstract}

\section{Introduction}

Excavator pose estimation systems are commercially available today [1] [2], but have not yet been widely adopted by the construction industry. This may be explained, in part, by two unmet consumer needs. First, excavator pose estimation systems are cost prohibitive for a large portion of the market, often requiring numerous sensors and expensive global positioning system (GPS) components. Second, the use of such sensor systems is limited to outdoor environments where GPS communication is available, due to the fact that GPS-based systems are susceptible to failure in areas with tall trees, in urban environments with tall buildings, and indoors [3]. Thus, the development of a low cost, ubiquitous excavator pose estimation system may help to fill a market need.

One potential approach to developing a low cost, ubiquitous excavator pose estimation system is to use optical cameras and fiducial markers as sensors. A fiducial marker, as described here, refers to a two-dimensional visual object having prescribed geometry and identification features to distinguish it from naturally occurring objects and other markers. Upon capturing video of such a marker, the camera's image can be analyzed to estimate the marker's pose, or location and orientation, relative to the camera [4]. Similarly, an object's pose can be inferred by placing a marker on the object of interest and viewing it with a camera. This method can be extended to the estimation of an excavator's pose by strategically placing markers on the machine's components and viewing them with cameras [5]. Marker-based sensor systems may offer such benefits over traditional systems as requiring fewer and less expensive sensors, and providing functionality in urban and indoor environments.

The remainder of this paper is organized as follows: Section 2 describes the technical problem and motivation for this research; Section 3 describes the iterative electromechanical design, prototype development, and evaluation process; and Section 4 draws the paper's conclusions and outlines directions for ongoing and future work.

\section{Research Motivation}

To begin, it is noted that the teeth of the excavator bucket represent the cutting edge of the machine, making 
tooth location and orientation the points of interest in most excavation activities. As previously stated, it is conceptually possible to determine the pose of a bucket's teeth by attaching a marker to the bucket and observing the marker with a camera. In the simplest case, tooth pose can be determined by placing a marker directly on the side of the bucket, aligned with the bucket teeth, as shown in Figure 1. Additionally, a camera can be aimed toward the marker with a known relationship to the jobsite. In such a manner, a kinematic chain can be established to relate tooth pose to the rest of the jobsite. If a model of the jobsite includes underground utilities, then the pose can also be determined relative to the utilities.

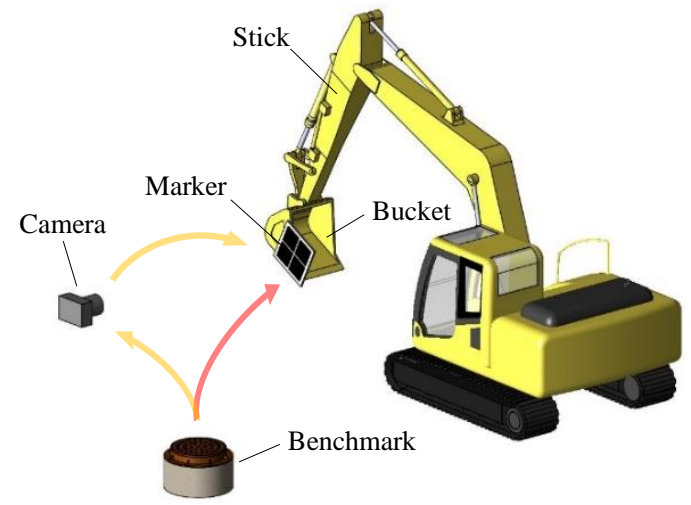

Figure 1. Conceptual marker-based sensor setup

However, there may exist benefits to moving the camera closer to the excavator cabin since it is a likely location for data processing and interfacing with the operator. In such close proximity to the marker, a second camera, with known relation to the first camera, can be added to help expand the sensor's vertical range. This cabinmounted camera setup may be accomplished through the use of one or more sentinel markers stationed nearby with known reference to the jobsite, as shown in Figure 2 Lastly, a third camera, having known relationships to the other cameras, may be added to view the sentinel marker.

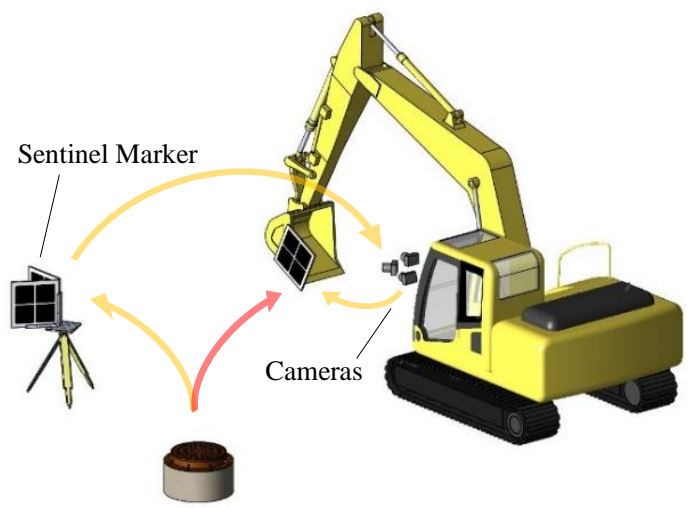

Figure 2. Multiple cameras with sentinel marker
However, the fact that the bucket penetrates the ground's surface while digging makes it impractical to place a marker near the teeth, as the ground would likely occlude the line of sight between the camera and marker. In fact, placement of a marker anywhere on the bucket may be impractical due to the likelihood of occlusion or damage during digging. Thus, an alternative method was needed to estimate tooth pose without placement of a marker directly on the bucket.

\section{Technical Approach}

\subsection{Four-Bar Linkage Design}

One solution for implementing marker-based tooth pose estimation without placing a marker directly on the bucket is to form a short kinematic chain involving the last two links of the excavator: the stick and the bucket. One marker can be used to provide an estimate of the stick's pose and another to estimate the bucket's rotation relative to the stick, as shown in Figure 3. The first marker, termed stick marker, is rigidly attached to the stick with a known relationship to the bucket's axis of rotation. The second marker, termed rotary marker, is attached at a location removed from the vicinity of the bucket. The rotary marker is constrained with one degree of rotational freedom and a known angular relationship to the bucket. If the bucket's geometry is also known, or measured onsite, then all necessary information is available to deduce tooth pose.
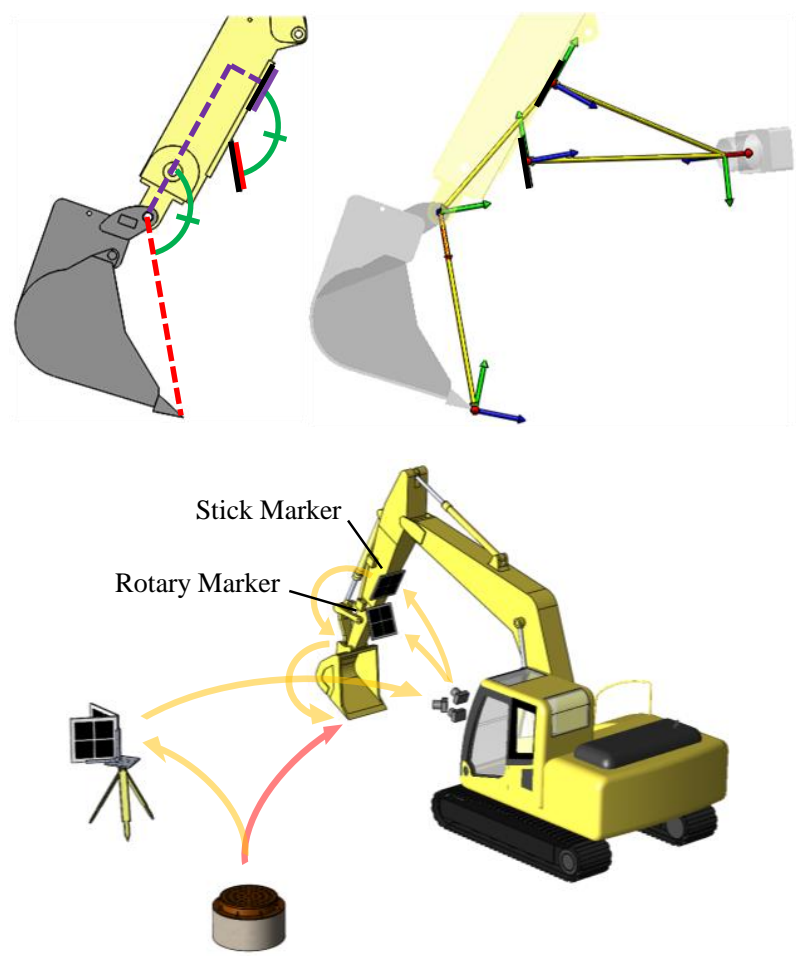

Figure 3. Marker-based kinematic chain and setup 
The four-bar linkage was one mechanism briefly explored for implementing such a system. An initial design for an experimental prototype was developed, as shown in Figure 4. The primary design objective involved transmitting the bucket's angle to a removed location and outputting the angle as a measurement of marker pose without permanently modifying the excavator. The design features a parallelogram four-bar linkage with strong magnets for attachment to the stick and bucket.
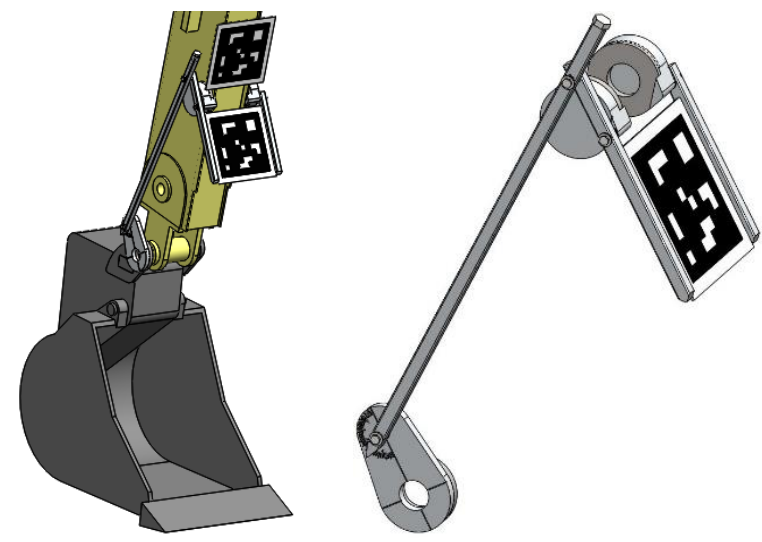

Figure 4. Four-bar linkage design

During the design process, it was realized that the operational range of an excavator bucket commonly exceeds 180 degrees, while the singularities inherent in a parallelogram four-bar linkage limit its functional range to less than 180 degrees. Although it may have been possible to avoid singularities through the use of a non-parallelogram four-bar linkage, such a mechanism would have introduced a nonlinear relationship between the bucket and marker, and was therefore avoided. Thus, the four-bar linkage design was discarded for an alternative free of singularities and nonlinearities.

\subsection{Synchronous Belt Design}

The synchronous belt and pulley system was identified as an alternative mechanism for transmitting bucket angle to a removed location without singularities or nonlinearities. In addition to providing continuous rotation, the belt and pulley system offers the benefits of low maintenance and gearing capabilities through the employment of different sized pulleys. A belt-driven prototype was designed and fabricated for the purpose of marker-based sensor experimentation, as shown in Figure 5. As with the four-bar linkage, the primary design objective involved accurately transmitting bucket angle to a marker at a removed location without permanently modifying the excavator. In order to accurately transmit bucket angle through the device, alignment between components was critical.

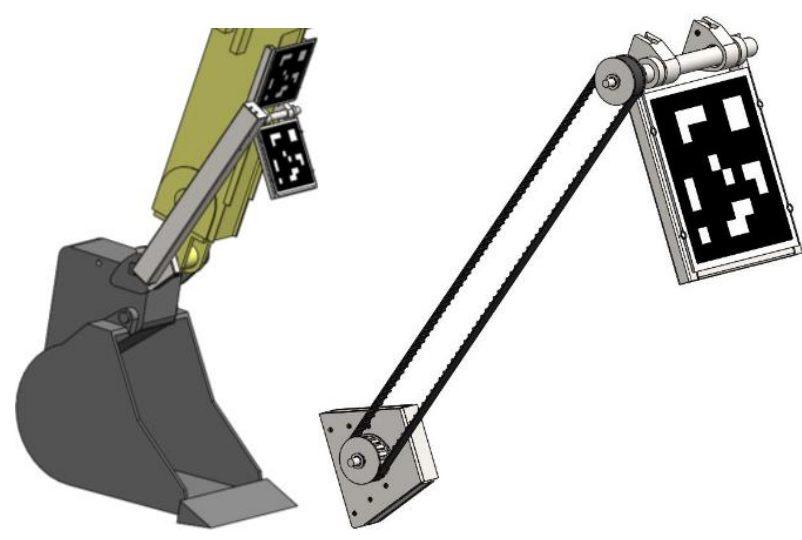

Figure 5. Synchronous belt design

At the base of the device is a bracket which clamps to the bucket through the use of set screws, thus providing sufficient anchoring for experimentation without permanently modifying or damaging the excavator. Before clamping, the bracket is aligned with the bucket's teeth and axis of rotation. Attached to the bracket is a synchronous pulley. During fabrication, the pulley is aligned with the bracket through the use of dowel pins and accurately located holes, as shown in Figure 6. The pulley then drives a synchronous belt, which transmits bucket angle to an upper pulley. The upper pulley is attached to a belt tensioning system which helps maintain accurate alignment between pulleys. The upper pulley also attaches to a shaft, which drives the rotation of the marker.

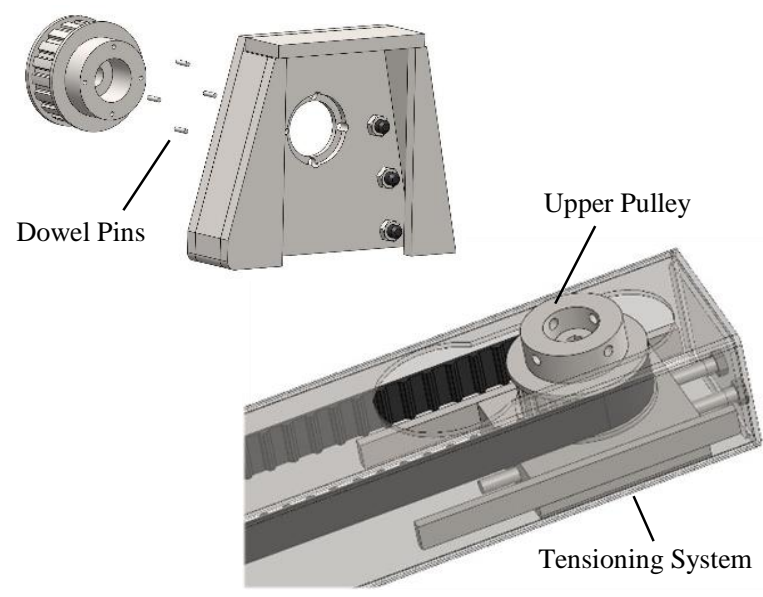

Figure 6. Pulley alignment methods

The synchronous belt prototype was fabricated, installed, and tested on a Caterpillar 430E IT Backhoe Loader, as shown in Figure 7. The experiment involved placing the backhoe in random poses, using the sensor system to estimate bucket tooth height, and comparing such estimates with ground truth measurements. Tooth height was selected as the measure of error because 
ground truth measurements could easily and accurately be ascertained using a rotary laser level and tape measure. The experiment's pass/fail criterion was set at 2.5 centimeters ( 1 inch) of absolute error. The markers used in the experiment were made from AprilTags, as developed by Olson [6].

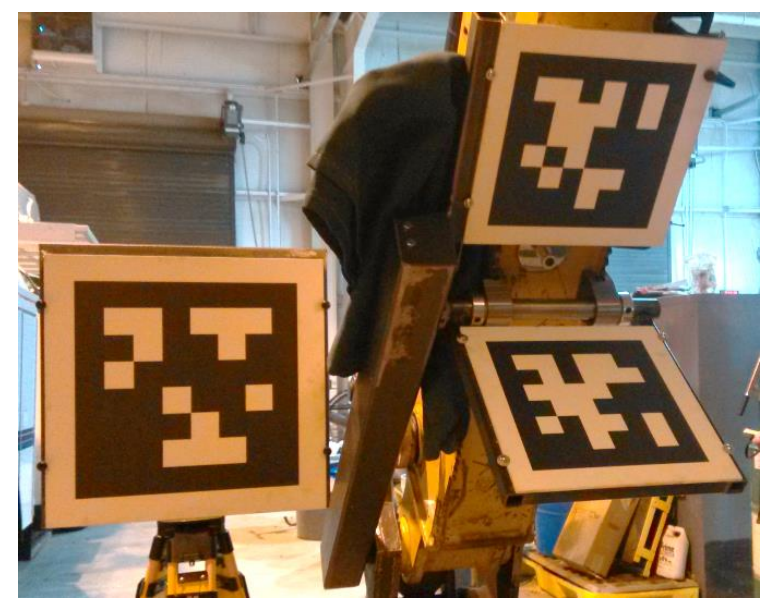

Figure 7. Installed synchronous belt prototype

Inferior camera calibration methods, software bugs, and lack of tag redundancy in the markers led to poor sensor performance, and the experiment was quickly terminated. After months of further camera and software development, the experiment was repeated. The results of the repeated experiment are shown in Figure 8, where it can be seen that all thirty estimates of tooth height fell within the allowable error limit of $+/-2.5$ centimeters $(+/-$ 1 inch).
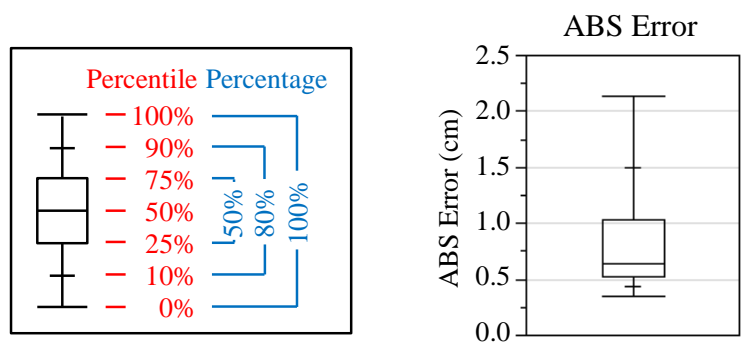

Figure 8. Synchronous belt experiment results

Despite the system's demonstrated ability to estimate tooth height, it was ultimately determined that the synchronous belt design was not practical for use on excavators. One concern was the hindrance of the bucket's clamping functionality by the presence of the device. Specifically, operators sometimes clamp objects between the bucket and stick for gripping purposes, but the locations of the markers in the synchronous belt design interfered with such action. Additional concerns included the device's lack of compatibility across different excavators, its need to be removed to change buckets, and its susceptibility to damage as a consequence of its high profile. An alternative design was sought to circumvent such issues.

\subsection{Bucket Linkage Design}

One design alternative having the potential for low mechanical profile and high compatibility across platforms was termed generalized mapping. Generalized mapping, as described here, involves the use of a calibration process to map a relationship between a bucket transmission component and bucket tooth pose. After establishing such a relationship, tooth pose can be inferred through measurement of the bucket transmission component.

One implementation of generalized mapping is bucket linkage generalized mapping. Bucket linkage generalized mapping entails placing a stick marker on the stick and a key marker on a bucket transmission linkage, as shown in Figure 9.

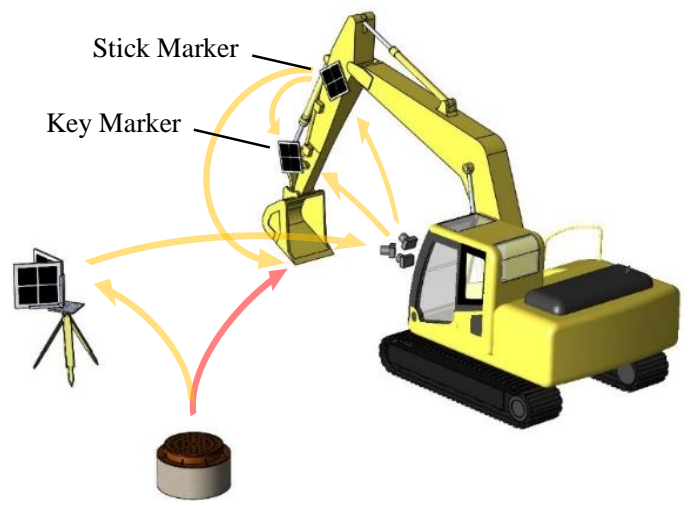

Figure 9. Bucket linkage design setup

A third marker is also placed on the side of the bucket and aligned with the bucket teeth for the duration of a calibration process, as shown in Figure 10. The system is then calibrated by moving the bucket through its range of motion and recording intermediate marker poses obtained by the camera system. The intermediate pose measurements provide a mapping which relates the relative transformation between stick marker and key marker to the transformation between stick marker and tooth pose. After the calibration process, the bucket marker is removed. The sensor system then functions by observing the stick and key markers, and mapping their relative pose to that of the bucket teeth.

One advantage of bucket linkage generalized mapping is that the placement of the stick and key markers is made nearly arbitrary by the calibration process. The markers can be placed on their respective components with little regard for alignment and location, as long as they are attached rigidly and with a clear line of sight from the cameras. Another advantage of the system is that 

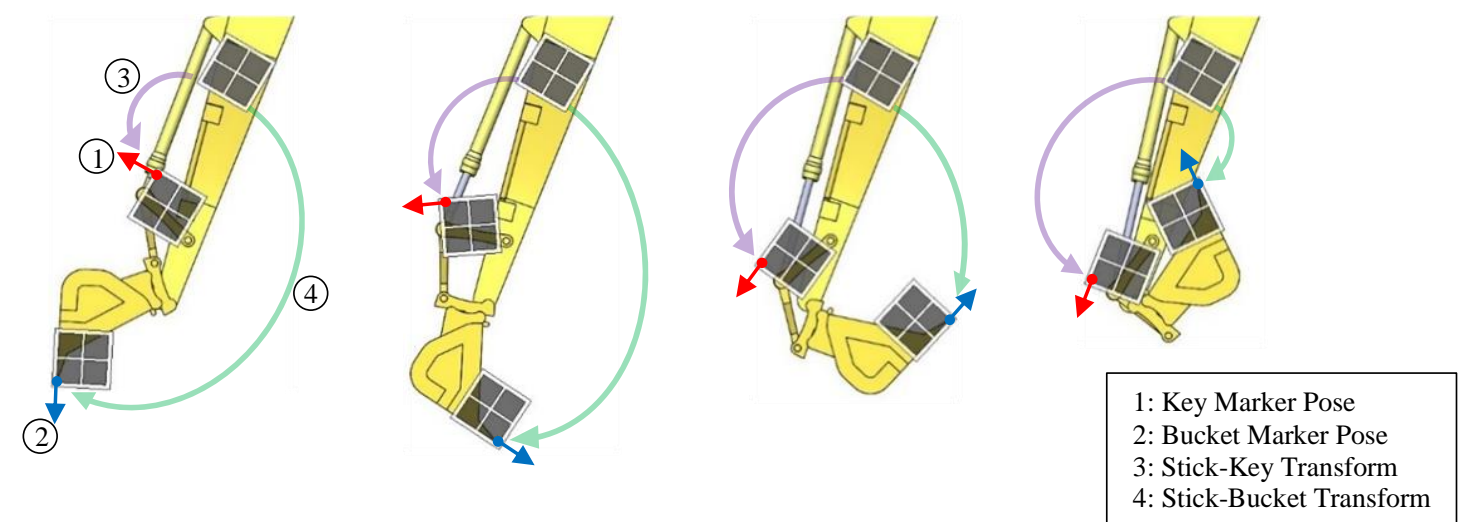

Figure 10. Calibration process for bucket linkage generalized mapping

its mechanical components are effectively flat, and thus have the potential for a low profile. Although the markers used for experimental testing had considerable surface area, it is not unreasonable to presume they could be made smaller through further sensor development. Lastly, the use of a calibration process allows for high compatibility between excavators. Effectively, the only criterion for compatibility is that the markers can be mounted on the machine.

A prototype of the bucket linkage generalized mapping system was installed and tested on a Caterpillar 430E IT Backhoe Loader, as shown in Figure 11. The markers used in the experiment were made from AprilTags, as developed by Olson [6]. The experiment involved calibrating the sensor system, placing the backhoe in random poses, using the sensors to estimate bucket tooth height, and comparing estimates with ground truth measurements obtained using a total station.
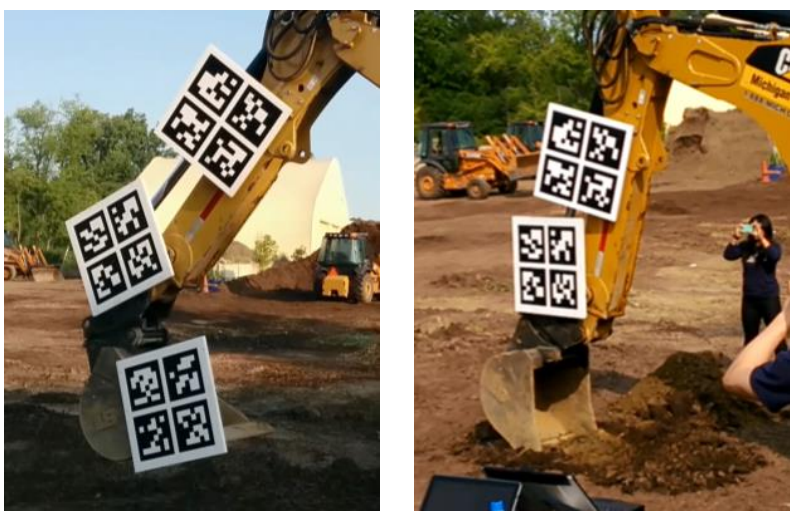

Figure 11. Installed bucket linkage prototype

The results of the initial experiment proved unsatisfactory. It was concluded that the key marker's resolution was too low to accurately determine tooth pose with the camera sensor system being used. It can be seen in Figure 12 that the key marker's radius of motion is considerably less than that of the bucket teeth. Thus, a small change in translation and rotation of the key marker corresponds to a large change in translation and rotation of the bucket teeth. Additionally, the resolution is further degraded by the nonlinear transmission of angle through the non-parallelogram four-bar linkage, which is evident in the total angles swept out by each marker. As a result, the error inherent in the sensor system is magnified by the key marker's low resolution. Mounting the key marker further from the linkage's axis of rotation may have helped to alleviate the resolution issue, but would likely have caused the marker to extend an impractical distance from the excavator. Thus, an alternative sensor was sought to provide a measure of bucket angle with higher resolution.

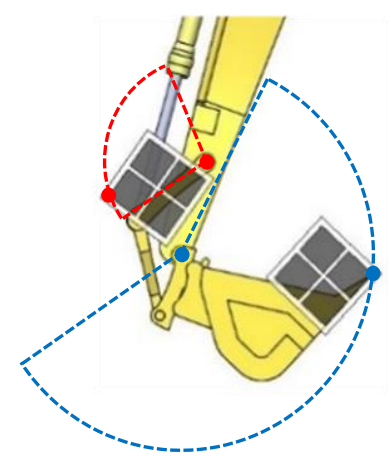

Figure 12. Comparison of radii and swept angles

\subsection{Cable Potentiometer Design}

The cable-driven rotary potentiometer was identified as an alternative sensor capable of measuring bucket angle with sufficient resolution. In addition to its resolution, the cable potentiometer was identified as an economical, robust sensor that can be installed on an excavator without permanently modifying the machine. Specifically, the cable potentiometer can be mounted on the bucket's hydraulic cylinder to provide a measure of stroke length, which can then be used to determine tooth pose through the generalized mapping approach. 
Cable potentiometer generalized mapping is accomplished much the same as bucket linkage generalized mapping. A calibration process is performed in which the bucket is moved through its range of motion and measurements of stroke length are mapped to the relative transformation between the stick marker and bucket teeth, as shown in Figure 13. After the calibration process, the marker is removed from the bucket. The system then functions by measuring stick marker pose and cylinder stroke length, and mapping such measurements to bucket tooth pose.

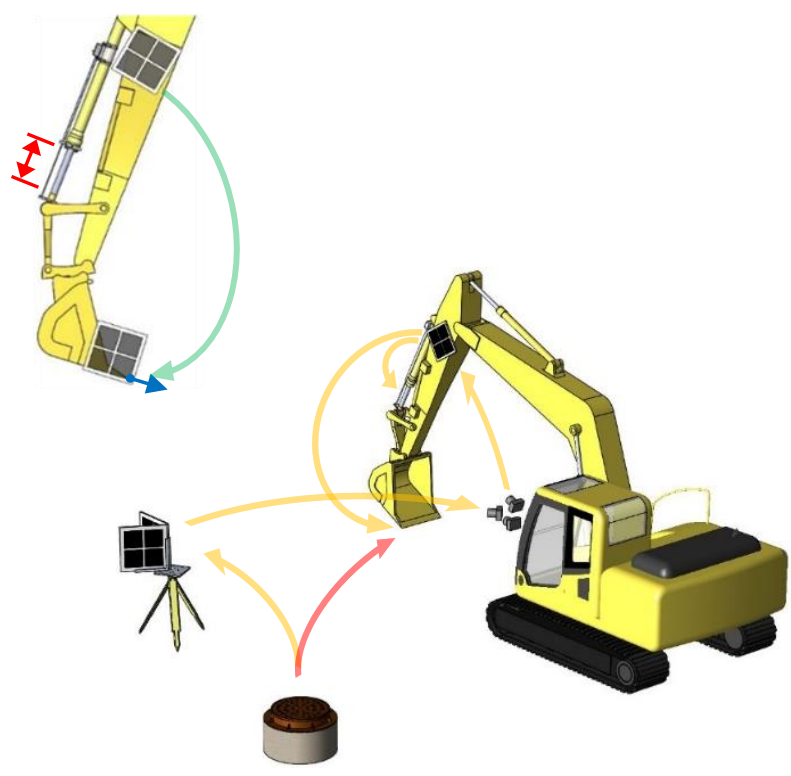

Figure 13. Cable potentiometer calibration and setup

A prototype was developed for the purpose of testing the cable potentiometer generalized mapping concept, as shown in Figure 14. The prototype attaches to the hydraulic cylinder through the use of clamps to avoid permanent modification of the machine. The device includes a cable potentiometer for measuring linear displacement, a microcontroller for signal conversion, a radio for wireless transmission, and a battery for power, all of which are mounted inside an enclosure for protection. The sensor's cable is connected to a fiberglass rod, which telescopes through a rigid pipe lined with plastic to reduce abrasion. If brought into contact with a foreign object, the rod's compliant properties allow it to temporarily deflect and later return to its natural shape. In the case of failure, however, the rod is expected to shear at the pipe and prevent the cable from being pulled out beyond its physical limit. In this way, the fiberglass rod protects the cable potentiometer by acting as a sacrificial component. Additionally, the rod's low strength also prevents damage to the hydraulic cylinder by limiting the forces transferred to the clamps.

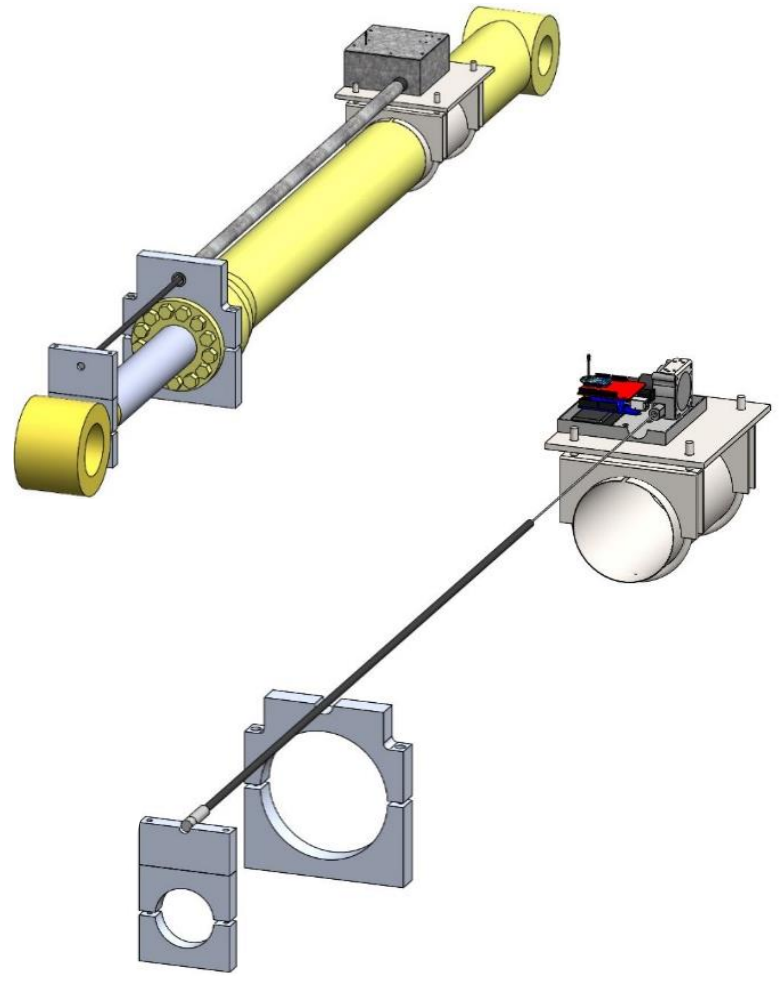

Figure 14. Cable potentiometer design

A cable potentiometer generalized mapping prototype was built, installed, and tested on a Caterpillar 430E IT Backhoe Loader, as shown in Figure 15. The markers used in the experiment were again made from AprilTags, as developed by Olson [6]. The experiment involved calibrating the sensor system, placing the backhoe in random poses, using the sensors to estimate bucket tooth height, and comparing estimates with ground truth measurements obtained using a total station. The experiment's pass/fail criterion was set at 2.5 centimeters ( 1 inch) of absolute error.

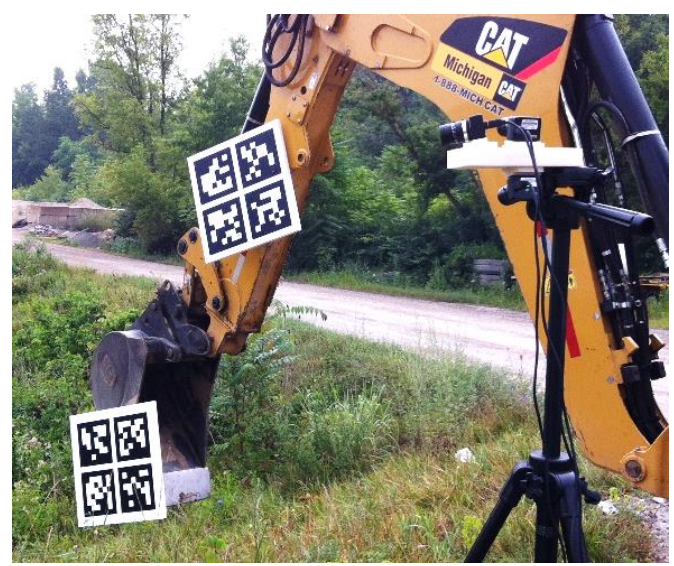

Figure 15. Installed cable potentiometer prototype 
A total of eight trials were conducted. For each trial, three components of tooth position $(x, y$, and $z$ ) were measured and compared with ground truth measurements, as shown in Figure 16. Of the twenty-four data points collected, only three points exceeded the pass/fail criterion of 2.5 centimeters ( 1 inch) of absolute error. Though the system's ability to estimate $x$-position only marginally met the pass criterion, the system's performance as a whole was deemed satisfactory.
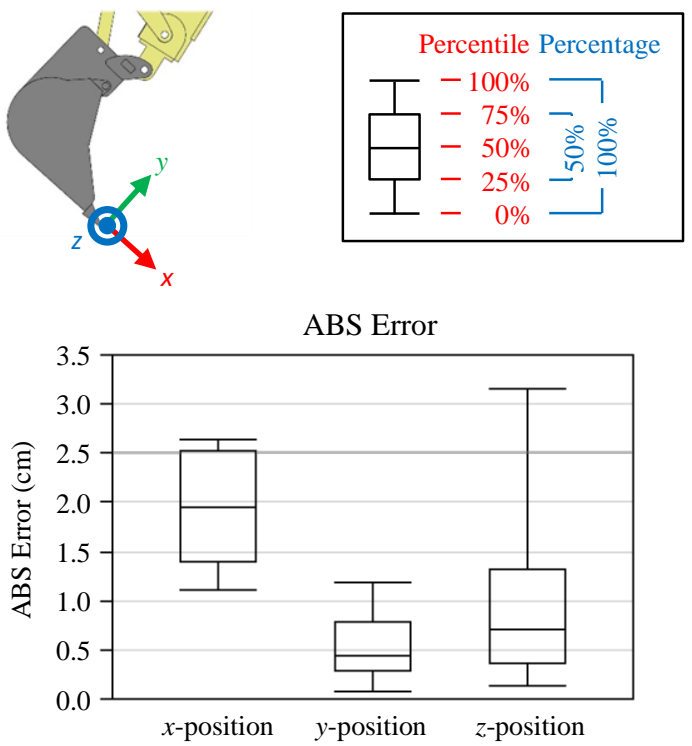

Figure 16. Cable potentiometer experiment results

The cable potentiometer generalized mapping prototype was also tested on a PC138USLC Komatsu Excavator operating on an active construction site, as shown in Figure 17. A computer screen was mounted in the cabin, providing a display of bucket height relative to a desired trench grade specified by the job plans. The system was used to assist the operator in trenching operations.
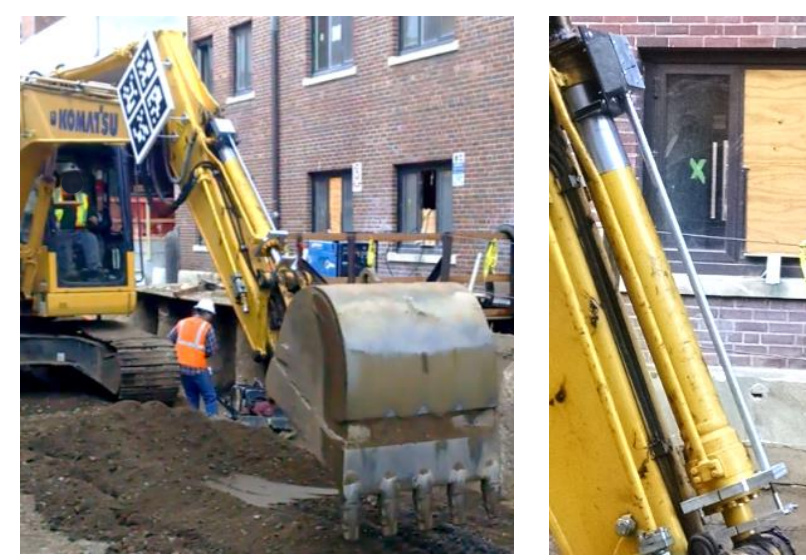

Figure 17. Cable potentiometer site testing
Shown in Figure 18 is a short section of trench in which the operator conducted a side-by-side comparison of traditional grading versus grading guided by the sensor system. The results of the comparison appeared satisfactory. After several days of function, the prototype incurred an impact to the fiberglass rod. The rod sheared off at the pipe opening as intended, and was thus considered a controlled failure. The failure, however, resulted in the termination of the experiment.

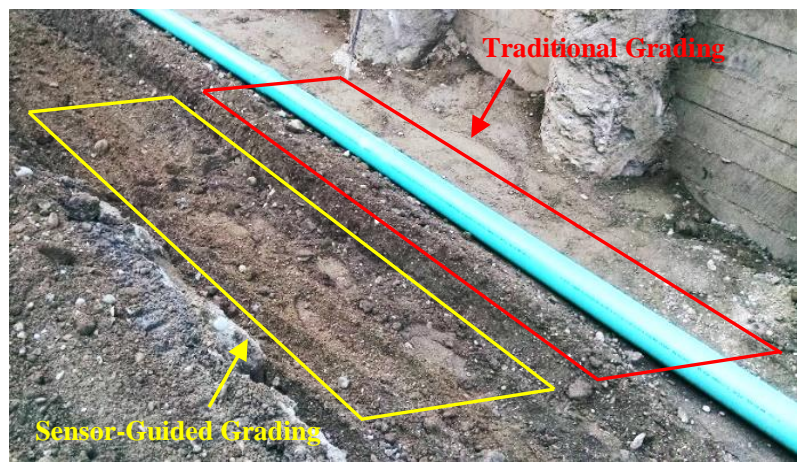

Figure 18. Traditional and sensor-guided grading

\section{Conclusions and Future Work}

Excavator pose estimation systems offer the potential to improve excavation safety and performance, but their widespread adoption is impeded by the high cost and limitations of traditional excavator pose estimation systems. A vision-based sensor system, comprised of fiducial markers and optical cameras, offers a potential low cost and ubiquitous alternative. However, because markers cannot be placed directly on an excavator's bucket for occlusion and durability reasons, a device is needed to transmit bucket motion and provide information necessary to deduce tooth pose.

Several design iterations were developed to address this problem, including a four-bar linkage design, a synchronous belt design, a bucket linkage generalized mapping design, and a cable potentiometer generalized mapping design. Prototypes were fabricated, installed, and tested. Promising experimental results suggest the feasibility of vision-based sensor technology for excavator pose estimation.

Marker-based sensors appear to offer a potential solution for the economical localization and pose estimation of articulated construction equipment, especially in environments with limited GPS capabilities, but their specific application and implementation remain unknown. Though several variations of marker-based pose estimation systems were demonstrated with reasonable success, a fully functional system has yet to be developed for use on actual construction sites. 
Future work is needed to identify the proper implementation of marker-based sensors in excavator pose estimation systems. For example, it is still unclear whether it is best to place cameras on an excavator in view of a jobsite, or on a jobsite in view of an excavator. One potential advantage of the former is the centralization of power, sensors, processors, and interface, while a potential advantage of the latter is the possibility of viewing multiple machines with the same camera. Also regarding implementation, it is unclear whether marker-based sensors should comprise the entire sensor system, or whether marker-based sensors and traditional sensors should be used to complement one another, and to what extent.

Another challenge is the potential for occlusion. Considering the busy nature of a construction site, it is not unreasonable to expect that a person, machine, or object might occasionally block the line of sight between a camera and marker. Thus it may be necessary to evaluate the severity of the concern and address it, possibly through the incorporation of redundancies. Similarly, as an excavator moves, it may prove challenging to keep markers in the field of view of the cameras. Again, one solution might exist in the use of multiple site-based cameras or multiple site-based markers for redundancy.

Lighting conditions may also pose a challenge, as low light levels can lead to marker detection loss. Thus, some form of active lighting may prove necessary for nighttime operation, and possibly even during dawn and dusk or in poorly lit indoor environments. Lastly, the harsh environment of the typical jobsite may present challenges for the system's components. Thus, a fully functional system will likely require considerable design efforts to ensure electrical and mechanical robustness. Such efforts are currently being pursued by the authors' research group.

\section{Acknowledgments and Disclosure}

This research was funded by the United States $\mathrm{Na}$ tional Science Foundation (NSF) via Grants CMMI1160937, CMMI-1265733, and IIP-1343124. The authors gratefully acknowledge NSF's support. The authors also thank Walbridge Construction Company, Eagle Excavation Company, and the University of Michigan Architecture, Engineering, and Construction (AEC) division for their support in providing access to construction equipment and job sites for experimentation and validation. Any opinions, findings, conclusions, and recommendations expressed in this paper are those of the authors and do not necessarily reflect the views of the NSF, Walbridge, Eagle Excavation, or the University of Michigan. Suyang Dong and Vineet R. Kamat have a significant financial and leadership interest in a start-up company named Perception Analytics \& Robotics LLC (PeARL), and are the inventors of technology involved in or enhanced by its use in this research project.

\section{References}

[1] Trimble, 2015, "Grade Control for Excavators," http://construction.trimble.com/products/machinecontrol/grade-control-for-excavators.

[2] Leica, 2015, "Machine Control, Digging Solutions," http://www.leica-geosystems.com/en/DiggingSolutions_63797.htm.

[3] Bossler, J. D., Campbell, J. B., McMaster, R. B., and Rizos, C., 2010, Manual of Geospatial Science and Technology, CRC Press, Boca Raton, FL.

[4] Feng, C., and Kamat, V. R., 2013, "Plane Registration Leveraged by Global Constraints for Context-Aware AEC Applications," Computer-Aided Civil and Infrastructure Engineering, 28(5), pp. 325-343.

[5] Feng, C., Dong, S., Lundeen, K. M., Xiao, Y., and Kamat, V. R., "Vision-Based Articulated Machine Pose Estimation for Excavation Monitoring and Guidance," Proc. International Symposium on Automation and Robotics in Construction (ISARC), ISARC.

[6] Olson, E., 2011, "AprilTag: A Robust and Flexible Visual Fiducial System," 2011 IEEE International Conference on Robotics and Automation (ICRA), IEEE, pp. 3400-3407. 